Licencia: Creative Commons (BY-NC-SA) 4.0 Internacional

\title{
Estrategia didáctica para la integración curricular de la biblioteca: creación de proyectos con Arduino y Scratch
}

Didactic Strategy for the Curricular Integration of the Library: Creation of Projects with Arduino and Scratch

Hazel Castro-Araya Universidad de Costa Rica, Costa Rica https://orcid.org/0000-0003-1875-5770

Daniela Esquivel-Calderón Sistema Nacional de Bibliotecas, Costa Rica https://orcid.org/0000-0002-6536-1814

Jairo Guadamuz-Villalobos Universidad de Costa Rica, Costa Rica https://orcid.org/0000-0002-0284-3656

Recibido:06 de noviembre de 2020

Aceptado:13 de agosto de 2021

Publicado: 25 de enero de 2022

\begin{abstract}
Resumen
El movimiento de software y hardware libre han socializado la tecnología permitiendo un acceso abierto a esta y reduciendo con ello la brecha digital. En el aula se ha encontrado en dichos movimientos la oportunidad para dinamizar las clases y los procesos de enseñanza y aprendizaje en general, no obstante, la biblioteca tiene un papel preponderante en dicha dinamización, pues este espacio de promoción de la información puede ofrecer al personal docente las herramientas tecnológicas y su conocimiento para su uso mediante la integración curricular y la planificación pedagógica. El presente artículo muestra la ejecución de una estrategia didáctica que le enseña a futuros profesionales en bibliotecología a crear proyectos para la integración curricular de sus unidades de información utilizando el software Scratch y los microprocesadores Arduino como apoyo a la planificación pedagógica. Muestra, además, los proyectos que el estudiantado desarrolló en dicha estrategia y la opinión de este en todo el proceso de enseñanza y aprendizaje experimentado.
\end{abstract}

\section{Palabras clave}

Tecnología educativa, Innovación, Arduino, Scratch, Planificación pedagógica, Integración curricular, Makerspace, Bibliotecas.

\section{Abstract}

The free software and hardware movement has socialized the technology allowing open access to it and thereby reducing the digital divide. In the classroom the opportunity has 
been found in these movements to boost classes and teaching and learning processes in general, however, the library has a preponderant role in such dynamization, because this space for the promotion of information can offer staff Teaching technology tools and knowledge of them for use through curriculum integration and pedagogical planning. This article shows the execution of a didactic strategy that teaches future professionals in library science to create projects for the curricular integration of their information units using Scratch software and Arduino microprocessors to support pedagogical planning. It also shows the projects that the students developed in said strategy and their opinion in the whole teaching and learning process experienced.

\section{Keywords}

Educational technology / Innovation / Arduino / Scratch / Pedagogical planning / Curriculum integration / Makerspace / Libraries.

\section{Introducción}

En la actualidad, la llegada de la Sociedad de la Información y del Conocimiento (SIC) provocada por la incidencia de las Tecnologías de la Información y Comunicación (TIC) en la cotidianidad del ser humano ha inducido cambios significativos en la educación primaria y secundaria. Las nuevas generaciones de estudiantes requieren un mayor control de las tecnologías con el fin de superar la brecha digital y poder avanzar en su preparación académica. Las bibliotecas, por su parte, deben buscar incidir en los procesos de enseñanza y aprendizaje, ofreciendo a su comunidad de usuarios (docentes y estudiantes) recursos y herramientas que les permitan tener espacios de aprendizaje ricos en el uso de tecnología, la resolución de problemas y el trabajo colaborativo.

Las bibliotecas escolares deben ofrecer espacios que permitan la interacción con hardware y software libre mediante el trabajo en equipo y, en complemento, el personal docente de diferentes disciplinas crea estrategias didácticas en conjunto con las personas profesionales en bibliotecología, con el fin de generar hechos educativos dentro de las instalaciones de la biblioteca.

El papel de la tecnología en la educación se puede ver reflejado factiblemente gracias a dicho movimiento libre, el cual posibilita el acceso abierto a herramientas tecnológicas sin restricciones, para así promover ambientes de colaboración y de búsqueda de nuevo conocimiento. Si bien es cierto, la biblioteca escolar permite el acceso a la información, promueve la lectura y da apoyo curricular; la evolución de las nuevas tecnologías y las 
demandas en la sociedad exigen una transformación de sus recursos y servicios. Ante esta realidad se destacan los espacios maker, los cuales pretenden promover la creatividad y la colaboración de la mano con el aprendizaje y la tecnología.

Por su parte, las universidades han ido creando estos mismos espacios no solamente para apoyar la labor docente en el aula, sino también para generar escenarios en los que futuros profesionales en bibliotecología tengan los recursos necesarios para diseñar por sí mismos estrategias didácticas que permitan la integración de la tecnología, el uso de la biblioteca y la materia impartida por las personas docentes en un mismo hecho educativo. Con este mismo fin, la Escuela de Bibliotecología y Ciencias de la Información (EBCl) de la Universidad de Costa Rica (UCR), a través del curso BI-5014 Herramientas y aplicaciones en la web para unidades de información educativas, propuso una estrategia didáctica que permite la integración de la tecnología educativa, el trabajo colaborativo y la resolución de problemas utilizando la biblioteca como espacio de convergencia entre estudiantes, profesores e información.

Para lograrlo, la EBCI trabajó en conjunto con el Programa de Tecnologías Educativas para el Aprendizaje (PROTEA), quienes ofrecieron apoyo didáctico y el uso de instalaciones y equipo propios de un espacio maker. PROTEA busca la generación de espacios para reflexionar, explorar y proponer nuevas formas de integrar y sacar provecho de los recursos tecnológicos. Esto se logra por medio de la colaboración con docentes que permiten experiencias interdisciplinares para el enriquecimiento de los procesos educativos.

El fin de dicho ejercicio fue acercar al estudiantado a la creación de una estrategia didáctica que pudiera ser utilizada en un aula de primaria y que, además, permitiera el uso de las instalaciones de la biblioteca, como un espacio maker donde participan profesores y estudiantes en la enseñanza y el aprendizaje de un contenido en particular. 


\section{Objetivos}

\section{a. Objetivo general}

Diseñar una estrategia didáctica para el desarrollo de habilidades en estudiantes de la Licenciatura en Bibliotecología con énfasis en Bibliotecas Educativas de la Universidad de Costa Rica para el uso de hardware y software libre (Arduino y Scratch) en el diseño de proyectos educativos para la integración curricular de la biblioteca.

\section{b. Objetivos específicos}

- Elaborar talleres para el manejo instrumental del software Scratch y el hardware Arduino para estudiantes de la Licenciatura en Bibliotecología con énfasis en Bibliotecas Educativas de la Universidad de Costa Rica.

- Apoyar a la población estudiantil en el diseño de un proyecto pedagógico basado en el uso del software Scratch y el hardware Arduino para la integración curricular en la biblioteca.

- Diseñar un espacio de socialización de los diferentes proyectos pedagógicos planteados por el estudiantado de la Licenciatura en Bibliotecología con énfasis en Bibliotecas Educativas de la Universidad de Costa Rica.

- Validar la estrategia didáctica desde la perspectiva del estudiantado de la Licenciatura en Bibliotecología con énfasis en Bibliotecas Educativas de la Universidad de Costa Rica.

\section{Referente teórico}

\section{a. Innovación y tecnologías educativas}

En la actual era digital los escenarios en que se presenta la información han tenido una evolución exponencial, originando innovaciones en los métodos y en los recursos para la enseñanza. Es de vital importancia entender las causas y qué se comprende por innovación y específicamente innovación educativa. Como lo menciona Margalef y Arenas (2006), "Innovación en relación con 'una invención', es decir, al proceso creativo por el cual dos o más 
conceptos existentes o entidades son combinados en una forma novedosa, para producir una configuración desconocida previamente" (p.3). Estos dos conceptos que mencionan las autoras son, por una parte, la educación y, por otra, la tecnología educativa, siendo la innovación un retomar y reinventar algo puesto en vigencia, pero que se busca entrelazar de forma novedosa.

La innovación y las tecnologías educativas son en la actualidad un factor incidente en el progreso de la ciencia pedagógica. Es evidente que, en este proceso, se encuentran aristas en relación con su aplicabilidad en el sector educativo, ya que la innovación didáctica en este campo se encuentra en rezago en relación con los avances de la tecnología. Tal desbalance entre educación-tecnología es manifiesto: en la formación del profesorado, en la ineficacia de los procesos de enseñanza y en la descontextualización de la práctica didáctica, entre otros. Como lo menciona Correa y Pablos (2009):

Aunque las ventajas y posibilidades de las TICs son muchas, también hay que considerar que la rentabilidad que se le puede sacar a la tecnología en el ámbito de la educación formal o de libre elección, choca precisamente con las limitaciones que imponen a su uso, modelos de enseñanza tradicionales excesivamente centrados en el profesor, en su papel y protagonismo en las aulas en el proceso de adquisición del conocimiento (p. 135).

Para Correa y Pablos (2009) la innovación y las tecnologías educativas son entornos donde la utilización de estos recursos permite la integración de las Tecnologías de la Información y la Comunicación [TIC] a las tareas cotidianas del aula, desencadenando un cambio en el modelo de enseñanza donde se descentraliza la participación del profesor; y se pone un mayor énfasis en el tratamiento de la información y cómo se obtiene la información. Es la innovación educativa, entonces, un involucramiento no solo en la praxis docente, sino un cambio en los procesos cognitivos y en el registro conductual de la población estudiantil. Las consecuencias de este modelo basado en la nueva praxis de innovación y tecnología, permite reelaborar el trabajo del alumnado, la actualidad del conocimiento, el enfoque curricular y la relación entre comunidad y centro educativo. 
Un estudio clásico sobre el concepto de innovación propone que innovar se relaciona con una idea creativa, o invención de un artefacto que se admira por su novedad, aisladamente de su aceptación, adopción o su uso (Zaltman, Duncan y llolbek, 1973). La tecnología como artefacto ya no es una novedad, sino que es parte integral de la cotidianeidad de la población estudiantil, y su innovación se centraría en la capacidad de utilizarlo de manera didáctica, aplicándolo tanto a las técnicas, como a los métodos de enseñanza.

La teoría que existe en la actualidad en relación con el binomio de innovación- educación, (Randi y Corno, 2000; Michavila, 2009; Sancho y Alonso, 2012; Sheeny, Ferguson y Clough, 2014) buscan el estímulo y la concientización en las tecnologías educativas, lo cual permite redefinir los procesos formativos, según los diferentes niveles educativos de la educación (preescolar, primaria, secundaria y la enseñanza superior). Partiendo así de una comprensión e influencia de las TIC en la sociedad actual, y considerando su uso en la cotidianeidad, como lo mencionan Paredes, Guitert y Rubia (2015):

Al pensar en la presencia de las TIC en la vida cotidiana, se sugiere que es posible otra relación con el conocimiento y el aprendizaje, y nos hace dudar sobre la forma en que están siendo introducidas las TIC en las escuelas, las políticas que lo soportan, los entornos de cultura de centro que lo favorecen, el origen de las innovaciones con las TIC, la importancia de los contextos de colaboración entre docentes para facilitarles y una valoración de los cambios acaecidos, que hace la innovación y la valoración de la misma igualmente necesarias (p. 102).

Estos autores reflexionan propiamente en las consecuencias positivas que conlleva buscar una innovación y el uso de la tecnología educativa y como, tal implementación tecnológica, consolida la competencia digital del profesorado, intensifica la comunicación entre el alumnado y la población docente; e incrementa, a su vez, la autonomía y el compromiso en las personas estudiantes en el proceso educativo. Es, por lo tanto, la innovación y la tecnología educativa un modelo que permite mejorar y flexibilizar los espacios, las condiciones de trabajo y la optimización en la práctica docente (Paredes et al, 2015). Según la propuesta en mención, el tema propiamente de la tecnología abarca no solo la aplicación de las TIC en las aulas; sino en el uso de metodologías que sean capaces de 
incorporar herramientas que profundicen los contenidos y que estos puedan ser usados de manera constante y productiva, por parte de la población estudiantil.

\section{b. Movimiento de software y hardware libre}

En el mundo de la tecnología educativa se destaca el movimiento libre como una herramienta que facilita al estudiantado adentrarse de manera autónoma en el software y hardware. Cabe destacar que el software se refiere a las partes intangibles como los componentes informáticos, o sea, programas, y el hardware corresponden a los componentes físicos ya sean placas, memorias, entre otros. En consideración a este movimiento, Stallman (2004) enfatiza en la libertad de ejecución, modificación y redistribución del software y hardware en dirección al progreso del nuevo conocimiento. Por otra parte, Valverde Chavarría (2005) menciona sobre la flexibilidad del software libre en la labor docente, dado que permite la colaboración y la reproducción sin restricción e influye en los ambientes de aprendizaje. En este contexto, la integración del movimiento libre en la educación es posible, gracias a que permite el acceso a las tecnologías de modo gratuito o de bajo costo.

Dentro de las herramientas del movimiento libre se resalta principalmente la placa Arduino. Herrero y Sánchez (2015) la definen como "sistema microcontrolador monoplaca, de hardware libre, de fácil uso y bajo coste, desarrollado inicialmente para facilitar el uso de electrónica en diseños artísticos e interactivos y la aplicación de esta por personas no expertas" (p.4). En el mismo sentido, Novillo-Vicuña et al (2018) expresan que el objetivo de Arduino es el "diseño y manufactura de circuitos electrónicos en circuitos impresos que incorporan un microcontrolador y el entorno de desarrollo para realizar la programación de cada placa de manera sencilla" (p.19).

En términos generales, Arduino nació en el 2005 en Italia respondiendo a una tecnología de software y hardware libre utilizando el lenguaje de programación $\mathrm{C}_{++}$. Originalmente, fue creada para ser accesible y ofrecer nuevos ambientes educativos para estudiantes que no cuentan con recursos económicos (Novillo-Vicuña et al, 2018). Por otra parte, los autores Herrero y Sánchez (2015) señalan que este tipo de plataforma proporciona escenarios donde la población usuaria tiene un mayor acercamiento a "la robótica, el control, la adquisición de datos, los diseños interactivos" (p.5) y se regeneran por medio de la imaginación y la creatividad en el desarrollo de nuevos proyectos. 
Asimismo, existe una amplia opción de placas Arduino que permiten la construcción de diferentes mecanismos y aparatos controlados por medio de la computación física o de manera autónoma, no obstante, la plataforma más popular es Arduino UNO R3. Otro aspecto para considerar es la Comunidad Arduino, la cual pretende ser un espacio de colaboración entre los usuarios para compartir las creaciones, tanto el boceto y el código, como la conexión eléctrica, facilitando por medio de las experiencias a otros usuarios no expertos el cómo crear nuevo conocimiento aplicable.

Como bien se mencionó anteriormente, Arduino utiliza un lenguaje de programación $\mathrm{C}_{++}$, este puede ser complejo para algunos usuarios en el sentido de que tiene comandos y códigos específicos para inicializar y poner en funcionamiento la placa, por tanto, existen otros entornos más amigables que permiten la codificación como Scratch for Arduino (S4A) para usuarios carentes de conocimientos en cuanto a la programación.

Para tener en claro S4A, es primordial comprender que es una modificación del programa Scratch en la cual adapta sus funciones para poder interactuar con la placa Arduino por medio de una programación en bloque. Específicamente, Scratch es un lenguaje de programación visual e iconográfico desarrollado por el Instituto de Tecnología de Massachusetts (MIT) en el 2002, con el objetivo de fomentar el pensamiento computacional bajo un movimiento libre y de código abierto. Su precedente es la aplicación Logo generado por Seymour Pappert en los años 60 (Tellez, 2016), en donde la población estudiantil genera un código de programación para mover una tortuga.

Según Valverde, Fernández y Garrido (2015) la premisa de mover objetos programables en Logo permanece con Scratch, por medio de "un gato color naranja que puede moverse, emitir sonidos o dibujar, entre otras posibles acciones que se diseñan a través de piezas preconfiguradas, que se encajan a modo de rompecabezas en una secuencia codificada según las intenciones del usuario" (p.4). Su lema es "Programa, Juega y Crea" por ello, se debe hacer hincapié en que es un programa para aprender jugando, tomando el error como una oportunidad de aprendizaje y de crear soluciones a problemas y no para entretener. La idea principal de Scratch es "crear historias interactivas, animaciones, 
juegos, música y arte" (Willging, Astudillo y Bast, 2010, p.4). En tal sentido, permite realizar animaciones a base de ladrillos movibles, es decir, por medio de una programación en bloque formando una secuencia de colores que representan las órdenes de programación indicadas a la placa Arduino.

\section{c. Tecnologías educativas en la biblioteca (Espacios Maker)}

La llegada de la tecnología en la educación no solo impactó los salones de clase, también impactó las bibliotecas. Al hacer referencia a las bibliotecas, de acuerdo con Orera (2008) se alude al papel ante la información, formación y desarrollo de cultura; a la tipología de las bibliotecas dirigida a la diversificación de usuarios; y a la evolución para satisfacer las necesidades de información.

Concretamente, cuando se apunta a las bibliotecas educativas, por lo general, se refiere a aquellas que giran en torno a los centros educativos, o bien, que forman parte de la mediación entre el usuario y la información de manera que contribuya en el proceso de enseñanza y aprendizaje. En este caso se hará alusión a las bibliotecas escolares, cuya presencia se demarca por ser un espacio de acceso a la información, de apoyo curricular y de fomento a la lectura; sin embargo, según Orera (2008) menciona que las bibliotecas han comenzado "un proceso de adaptación a estos cambios, con la incorporación de nuevos soportes en sus colecciones, la aplicación de nuevas tecnologías a los procesos de gestión y servicios a los usuarios" (p.20). En relación con lo indicado anteriormente, la biblioteca tradicional debe cambiar junto a la evolución tecnológica, buscando ofrecer nuevas formas de acceso a la información por medio de los dispositivos electrónicos, buscando posicionarse como un elemento vital en la comunidad de usuarios a la que sirve.

Por su parte, el tema de las tecnologías educativas está relacionado con aparatos o dispositivos que están conectados a una red, sin embargo, este término se remonta a otros dispositivos como el lápiz o el papel, o incluso la imprenta, en donde se da una invención de nuevas herramientas para solucionar problemas prácticos. En términos de tecnología educativa la autora Paau Cho (2009) cita que es:

La organización integrada de personas, significados, conceptualizaciones, procedimientos, accesorios simples y complejos, adaptados para ser utilizados para 
la elaboración, implementación y evaluación de programas y materiales educativos como procesos y productos que promuevan el aprendizaje contextualizado de manera libre y creativa (p.59).

En otras palabras, esta pretende ser "aquella que se usa para educar y se usa cuando se quiere educar a la gente de una manera innovadora" (Paau Cho, 2009, p.15). Bajo esta perspectiva se destacan las TIC, determinadas por ser "dispositivos o instrumentos, métodos y técnicas para el logro, el tratamiento, el almacenamiento y la transmisión de información" (Seas, 2016, p.290), las cuales promueven ambientes educativos de apropiación de tecnología y favorecen la creatividad, la innovación y la accesibilidad.

Todo esto puede ser aplicable dentro de las bibliotecas educativas, en el sentido de que actualmente disponen de dispositivos como computadoras, televisores inteligentes, tabletas, entre otros, acompañados de conexión a Internet, los cuales colaboran en su gestión, promoción y servicio. Al incluir en su labor bibliotecaria la tecnología es importante que las unidades de información se mantengan en constante actualización y que, al mismo tiempo, integren nuevas herramientas para satisfacer las demandas educativas.

Una de las tendencias más destacadas en las bibliotecas son los espacios maker, o en inglés, makerspace, dado que se toman como entornos tecnológicos que promueven en los usuarios crear, producir y compartir el trabajo realizado. Pritchard (2014) menciona que se caracterizan por contemplar los intereses de la población usuaria, las herramientas, el equipo tecnológico, el aprendizaje, la creatividad y el prototipado. Estos espacios no se restringen a una lista específica de materiales, estos pueden variar de acuerdo con el contexto y las necesidades de la comunidad usuaria; sin embargo, algunas de las herramientas más comunes entre los espacios maker son impresora 3D, kits de robótica educativa, máquinas de coser, cortadora láser, entre otras.

Bajo esta perspectiva, la biblioteca no pasa a ser un laboratorio informático, sino, que evoluciona a ser un espacio para generar nuevo conocimiento de la mano con la tecnología y la investigación. Es importante recalcar que el motor del makerspace es la necesidad de la persona usuaria, es decir, se brindará el espacio y las herramientas para que el usuario realice su proyecto creando la cultura maker, o bien, la cultura de hazlo tú 
mismo. Según Yockey y Donovan (2015) esta cultura pretende activar las manos de manera que la población usuaria realice un producto. Este proceso de activación comienza cuando el usuario identifica un problema, busca una solución utilizando su creatividad e imaginación y luego la diseña utilizando las diversas herramientas con la que cuenta el espacio maker.

El provecho de un makerspace en las bibliotecas educativas se ve dimensionado en la instauración de un ambiente de trabajo, de colaboración y multidisciplinar, en donde el proceso de enseñanza y aprendizaje se experimenta, imagina, crea, comparte, diseña y soluciona, generando nuevo conocimiento y aprendizaje significativo en la comunidad estudiantil.

\section{d. Integración curricular de la biblioteca}

Las escuelas y colegios en Costa Rica poseen en su organigrama, en la mayoría de los casos, bibliotecas escolares que brindan apoyo bibliográfico al personal docente y administrativo y a la comunidad estudiantil. Este apoyo se brinda más allá de la oferta de una colección y sus respectivos servicios y trasciende la necesidad de coordinar esfuerzos entre profesores y profesionales en bibliotecología para el desarrollo de clases, eventos académicos y promoción de lectura. La integración curricular de la biblioteca coloca a las unidades de información como co-protagonistas en los procesos de enseñanza y aprendizaje, de forma tal que el personal docente puede ver las bibliotecas no solo como un lugar de consulta, sino también como un espacio para el desarrollo de clases; y al profesional en bibliotecología puede vérsele también como un segundo docente que apoya la planificación de las estrategias didácticas y actúa en las clases como un segundo facilitador.

Según Castrillo y Méndez (2015), la integración de la biblioteca al currículo escolar "se puede lograr estableciendo una relación estrecha entre la biblioteca escolar y el proyecto educativo institucional, con el fin de acompañar los proyectos de la institución y al mismo tiempo ser un espacio complementario de interacción con los diversos materiales educativos" (p. 36). Es decir, la biblioteca debe ofrecer materiales útiles para la generación de hechos educativos y, además, debe estar pendiente de que estos recursos ofertados sean pertinentes no solo a los objetivos de aprendizaje de cada curso, sino también a las tendencias predominantes en su contexto, como los son en la actualidad el trabajo colaborativo, la resolución de problemas y el prototipado. 
La integración curricular de la biblioteca, por tanto, requiere de profesionales en bibliotecología que generen espacios de interacción entre docentes, estudiantes y conocimiento y que, además, tengan la capacidad de proponer estrategias didácticas para dicha integración en conjunto con el personal docente que así lo requiera. Es oportuno, entonces, que en la actualidad los profesionales en bibliotecología manejen tecnologías educativas para la resolución de problemas, el trabajo colaborativo y el prototipado.

\section{e. Planificación pedagógica}

EI MPPE, citado por Altuve, define la planificación pedagógica como "un proceso continuo y sistémico de construcción colectiva, con el propósito de organizar, diseñar, implementar, direccionar, coordinar, evaluar y sistematizar acciones y actividades que permitan el desarrollo del proceso de aprendizaje garantizando la transformación educativa" (2016, p.132). Es decir, se trata de un trabajo colaborativo e interdisciplinario que permite el diseño de estrategias didácticas, su ejecución y su respectiva evaluación, para generar procesos de enseñanza y aprendizaje que logren el cumplimiento de los objetivos planteados en el aula.

Desde la biblioteca, dicha planificación pedagógica va de la mano con la integración curricular de la biblioteca, por lo que requiere de un trabajo continuo entre el personal docente y la persona profesional en bibliotecología encargada de la unidad de información. Es indispensable que los futuros profesionales de la información cuenten con herramientas necesarias para participar en procesos de planificación educativa con el profesorado de cualquiera de las asignaturas que se imparta en su institución. La presente investigación busca dotar a la comunidad estudiantil de la $\mathrm{EBCl}$ de las capacidades necesarias para gestionar dicha planificación y lo hace justamente con un ejercicio de planificación pedagógica llevado a cabo por un equipo interdisciplinario conformado por miembros de la EBCl y el PROTEA. Para dichos efectos se incluyó la definición de objetivos claros de aprendizaje, actividades necesarias para alcanzar dichos objetivos (a desarrollarse dentro y fuera del aula), el seguimiento por parte de los facilitadores docentes y la evaluación de todo el proceso planificado. Además, el estudiantado, en el proceso de realizar su propia planificación pedagógica, debió construir prototipos de los proyectos que planeaban enseñar con sus estrategias didácticas propuestas. 


\section{Metodología}

Como estrategia metodológica se utilizó el ABP (Aprendizaje Basado en Proyectos) el cual es un modelo o paradigma educativo basado en la participación, donde el estudiantado tiene como eje disciplinar la intención estratégica de planificar, implementar y evaluar (Díaz, 2015). Este proceso es una búsqueda de fines concretos hacia un producto acabado y permite la movilización constante del conocimiento. Por otra parte, también se utilizó la encuesta como técnica de recolección de información para evaluar el uso didáctico de Arduino en el estudiantado participante. Así mismo, se evaluó la experiencia, su aplicación y el interés por este tipo de herramientas en futuras recomendaciones en la enseñanza de esta tecnología.

La información se recolectó por medio de un instrumento con preguntas abiertas y notas de campo durante la elaboración de los talleres de Arduino y Scratch, durante el proceso de elaboración de las estrategias y el día de la exposición de los proyectos. La información recopilada a lo largo de la aplicación de la estrategia didáctica fue procesada mediante un análisis e interpretación de datos cualitativos de forma descriptiva, atendiendo los tres temas principales de las categorías: la percepción de la experiencia con el diseño del material didáctico elaborado con Arduino; el proceso de enseñanza - aprendizaje de la estrategia pedagógica; y los aportes de la experiencia en el desarrollo de habilidades en la población estudiantil.

El ABP como estrategia metodológica, es un recurso que permite generar el espacio para la resolución de problemas, planteando inquietudes teóricas o prácticas en la población estudiantil. Esto con el fin de que se encuentre una comprensión en los temas asignados a través de actividades en conjunto. Al respecto menciona Cobo y Valdivia (2017) "El aprendizaje basado en proyectos es una metodología que se desarrolla de manera colaborativa que enfrenta a los estudiantes a situaciones que los lleven a plantear propuestas ante determinada problemática" (p. 5).

La aplicación del ABP como modelo de aprendizaje tiene como característica central el compromiso constante de la población docente, pero en este caso, el profesor o profesora 
participante tiene la función de guía y orientador en el proceso pedagógico; estimulando constantemente el aprendizaje colaborativo y participativo por medio de una evaluación real. Como lo menciona Martí, Heydrich, Rojas y Hernández (2010) "El proyecto no se enfoca solo en aprender acerca de algo, sino en hacer una tarea que resuelva un problema en la práctica. Una de las características principales del ABP es que está orientado a la acción" (p. 4).

En el proceso educativo, la aplicación del ABP permite el desenvolvimiento y aplicación de actos en torno a la autorregulación, dirección, y estimulación en el aspecto colaborativo como individual (Rodríguez, Vargas y Luna; 2010). Esto aplicaría tanto para la población estudiantil como para el profesorado, siendo uno de sus propósitos el incitar pedagógicamente al compromiso y a la responsabilidad en un involucramiento de las partes; estas habilidades son ejes del desarrollo cognitivo en formación de actitudes esenciales para la vida, optimizadas en el proceso de las ABP. Al respecto mencionan Rodríguez et al (2010):

Con esta estrategia, el aprendizaje es propositivo y autónomo, ya que el estudiante aprende a medida que investiga las soluciones a los problemas que se han formulado. Algunos aspectos del $\mathrm{ABP}$ se fundamentan en problemas intencionalmente mal estructurados (ill-structu-red), situaciones auténticas y oportunidades para desarrollar autonomía y responsabilidad (p.5).

El tipo de aprendizaje promovido en el ABP permite una participación por parte del estudiantado $\mathrm{y}$, de esta forma, aplica estrategias para obtener un producto final. En el proceso de creación se llevan a cabo tres ejes investigativos: la identificación de un mercado u objeto de estudio, investigación significativa sobre la temática, plan de gestión, diseño y elaboración del producto (Rodríguez et al, 2010).

Este proceso mencionado permite alcanzar resultados planteando problemas reales, los cuales se realizan a través de las propuestas originales de las personas participantes; siendo un método más efectivo y útil, tanto en términos de pedagogía como de procesamiento de información. Rodríguez et al (2010) menciona los datos obtenidos en el estudio realizado por Sousa (1995): 
En estudios realizados se ha comprobado que la retención del conocimiento adquirido después de 24 horas en un estudiante es de 5\% para clases magistrales, 50\% para discusión en grupo, $75 \%$ para experiencias prácticas y $90 \%$ por enseñar a otros (p. 4).

El ABP tiene un sistema sólido a partir de una generación de conocimientos en los que el estudiantado se dedica a llevar a cabo un aprendizaje basado en la práctica. Siendo la información obtenida o recibida un fluir constante de transformaciones, los cuales se orientan a la investigación y a la solución de problemas.

Por otra parte, la metodología utilizada para esta estrategia didáctica es de tipo cualitativo, contribuye, por su parte, con el marco de promoción de uso de las tecnologías, con el fin de desarrollar habilidades en los estudiantes de la Licenciatura en Bibliotecología con énfasis en Bibliotecas Educativas de la Universidad de Costa Rica. Se trabajó con un total de 16 participantes, 12 mujeres y 4 hombres, sin embargo, solamente 10 de ellos respondieron al instrumento. Para la identificación anónima de cada una de las opiniones recolectadas se utilizarán los códigos PEB01 al PEB10.

Finalmente, el estudiantado en ejercicio elaboró en su proyecto una estrategia didáctica unificando Arduino y Scratch, buscando la integración curricular y resaltando el papel de la biblioteca en el proceso de enseñanza y aprendizaje. Para ello, se utilizó tanto apoyo técnico como pedagógico aludiendo a talleres y sesiones en las que debían diseñar y crear un proyecto pedagógico, basado en alguno de los ejes temáticos incluidos en los programas de estudio del MEP (Ministerio de Educación Pública). Cada una de las creaciones elaboradas por el estudiantado se presentó en una actividad de socialización titulada "Feria de estrategias didácticas con microprocesadores Arduino".

\section{Resultados}

\section{a. Taller de programación en Scratch y uso de placas Arduino}

Para el cumplimiento del objetivo específico 1 se introdujo al estudiantado en el mundo de la programación en bloque utilizando Scratch y luego al uso del microprocesador Arduino UNO R3 con Scratch for Arduino (S4A) mediante talleres teórico-prácticos. Cada uno de los talleres se enfocó en el uso de las herramientas como un medio de aprendizaje y en el 
método didáctico del ABP. Del mismo modo, se utilizó el error como un medio de aprendizaje y el aprender a pensar de mejor manera la tecnología.

Respecto al taller de uso del software Scratch, los estudiantes realizaron una serie de retos para comprender que cada acción corresponde a una secuencia de instrucciones como estrategia de sensibilización para entender la programación en bloque. Este se realizó el miércoles 8 de mayo del 2019 en el Laboratorio de la Facultad de Educación de la Universidad de Costa Rica. Su duración fue de 3 horas con 50 minutos y contó con un receso.

Se asignaron tres retos. El primer reto fue dibujar un piano y recitar canciones con el teclado numérico de la computadora. El segundo reto fue hacer un cuento corto en donde los personajes se movieran e interactuaran considerando los tiempos entre las acciones. El tercer y último reto fue realizar un juego. Las funciones que se utilizaron para los retos fueron eventos, control, sonidos, apariencia y operadores.

Durante los retos, el estudiante fue guiado durante el proceso y atendido de manera que el mismo solucionara sus problemas sin la necesidad de que se brinde la respuesta inmediata, fomentando la creatividad y originalidad en las animaciones. Al finalizar el taller de Scratch se compartieron los proyectos creados y se comentaron las experiencias durante el taller y su vinculación con una estrategia didáctica, servicio o recurso en las bibliotecas educativas.

En cuanto al taller del hardware Arduino se dividió en dos sesiones. En la primera sesión se explicó sobre la placa y sus secciones y, posteriormente, los estudiantes realizaron una serie de ejercicios guiados con el uso del programa Tinkercad, tales como encender y apagar un led para comprender el circuito eléctrico respecto a la corriente, voltaje y resistencia. Esta actividad se realizó el miércoles 15 de mayo del 2019 en la sala SITEA de PROTEA en la Facultad de Educación de la Universidad de Costa Rica. Su duración fue de 3 horas con 50 minutos y contó con un receso.

Para la segunda sesión, el estudiantado continuó con otros ejercicios conectando diferentes dispositivos de Arduino como un botón, un buzzer, un potenciómetro y un servo motor; luego planearon su proyecto como estrategia didáctica vinculada a un plan de 
estudios del MEP. Esta se realizó el miércoles 22 de mayo del 2019 en la sala SITEA de PROTEA en la Facultad de Educación de la Universidad de Costa Rica. Su duración fue de 3 horas con 50 minutos y contó con un receso.

\section{b. Asignación de proyecto "Diseño de una estrategia de integración curricular desde la biblioteca con Scratch y Arduino"}

Para el cumplimiento de los objetivos específicos 2 y 3 se le asignó a la población estudiantil un proyecto de investigación como parte de la evaluación general del curso. En dicho proyecto, las personas estudiantes eligieron un contenido teórico entre las temáticas establecidas por el MEP, diseñaron una estrategia didáctica basada en los contenidos electos en la cual debían hacer uso de sopas de letras, infografías, crucigramas, formularios en línea o tiras cómicas.

Además, dicha estrategia didáctica debía incluir la construcción de un invento que ilustrara los contenidos electos, haciendo uso de un microprocesador marca Arduino, con el fin de que cada grupo de estudiantes propusiera el uso de microprocesadores como apoyo al proceso de enseñanza y aprendizaje. Adicionalmente, el proyecto debía incluir una estrategia de promoción con material gráfico y audiovisual que invitara al público meta a participar de la estrategia.

Finalmente, las propuestas creadas por cada uno de los grupos de estudiantes se socializaron en un evento titulado Feria de estrategias didácticas con microprocesadores Arduino y se realizó el miércoles 03 de julio de 2019 en las instalaciones de la Escuela de Bibliotecología y Ciencias de la Información de la Universidad de Costa Rica. (Figura 1) 


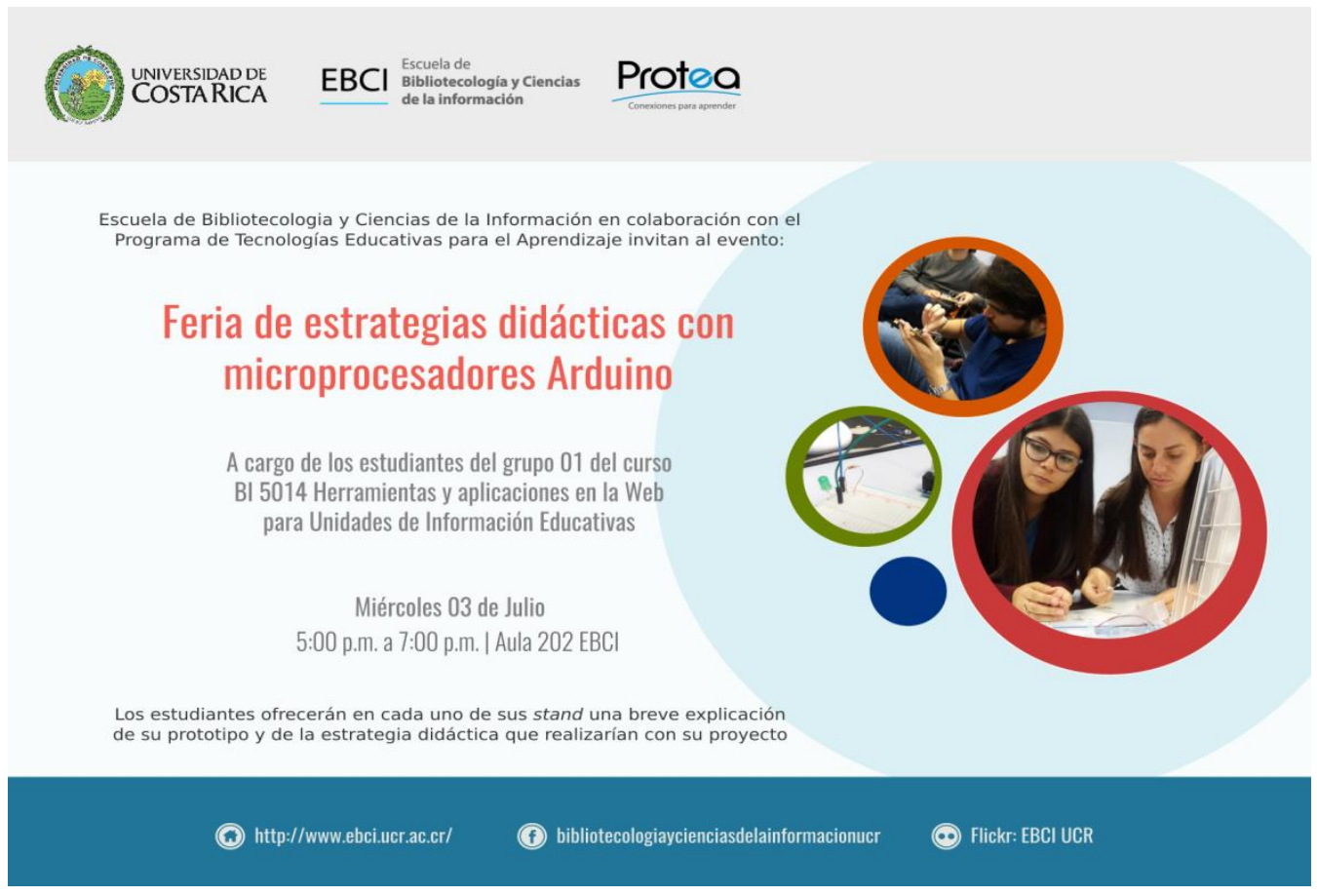

Figura 1: Afiche de promoción del evento de socialización

Fuente: Elaboración propia de las personas autoras, 2021.

Durante el evento se contó con la presencia de cada uno de los grupos de estudiantes con sus proyectos creados y con la visita de estudiantes interesados, personal docente y administrativo de la Escuela de Bibliotecología y del pasante Dr. Antonio Lorca Marín, proveniente de España.

\section{c. Seguimiento a proyectos propuestos}

Desde la fecha de asignaciones de los proyectos, hasta el día de su exposición en el evento de socialización, el estudiantado contó con dos meses para la planificación de su estrategia, el diseño de su prototipo con microprocesadores marca Arduino y la creación del material gráfico y audiovisual para la promoción de su estrategia. En todo este proceso, se tuvo la asesoría del profesor del curso Jairo Guadamuz Villalobos y la instructora del taller de Scratch Daniela Esquivel Calderón, así como Sofía Fonseca, asistente de PROTEA.

Este seguimiento incluyó la resolución de consultas, la validación de las ideas propuestas para cada uno de los prototipos diseñados y el acompañamiento durante la construcción de cada uno de ellos. La población estudiantil pudo tomar decisiones informadas sobre qué 
elementos (luces, sensores, motores, entre otros) elegirían para su uso en el microprocesador, gracias a la información recibida en el taller de uso de procesadores marca Arduino, así como a las consultas realizadas durante el proceso de seguimiento.

Los procesadores, sensores, luces y motores con los que trabajaron cada uno de los grupos de estudiantes fueron facilitados, en condición de préstamo, por PROTEA, por lo que al finalizar cada proyecto los elementos utilizados se devolvieron a las instalaciones del Programa.

\section{d. Proyectos diseñados por la población estudiantil}

Tras la realización de cada una de las etapas del proyecto, se presentaron en el evento de socialización un total de seis estrategias didácticas basadas en uso de microprocesadores marca Arduino. Entre los proyectos presentados se halla un prototipo que muestra las diferentes partes del ciclo de metamorfosis de una mariposa, con el fin de apoyar la enseñanza de las etapas de desarrollo de los seres vivos (Figura 2).

Otro de los prototipos diseñados aborda el tema de las energías limpias y renovables mediante una maqueta que reproduce la generación de energía eléctrica a través de un molino en movimiento, gracias al aprovechamiento del cauce de un río. En dicha representación a escala puede apreciarse el molino de un trapiche girando para moler caña de azúcar y producir dulce de tapa (Figura 3).

En la Feria también se expuso un proyecto relacionado con la temática del sistema solar. Dicho prototipo consistió en una representación a escala del sistema planetario, en donde cada uno de estos planetas realiza movimientos cortos para ilustrar la órbita que dibuja en su recorrido alrededor del sol (Figura 4). 
Bibliotecas. Vol. 40, №1, enero - junio, 2022. EISSN: 1659-3286

URL: http://www.revistas.una.ac.cr/index.php/bibliotecas/index

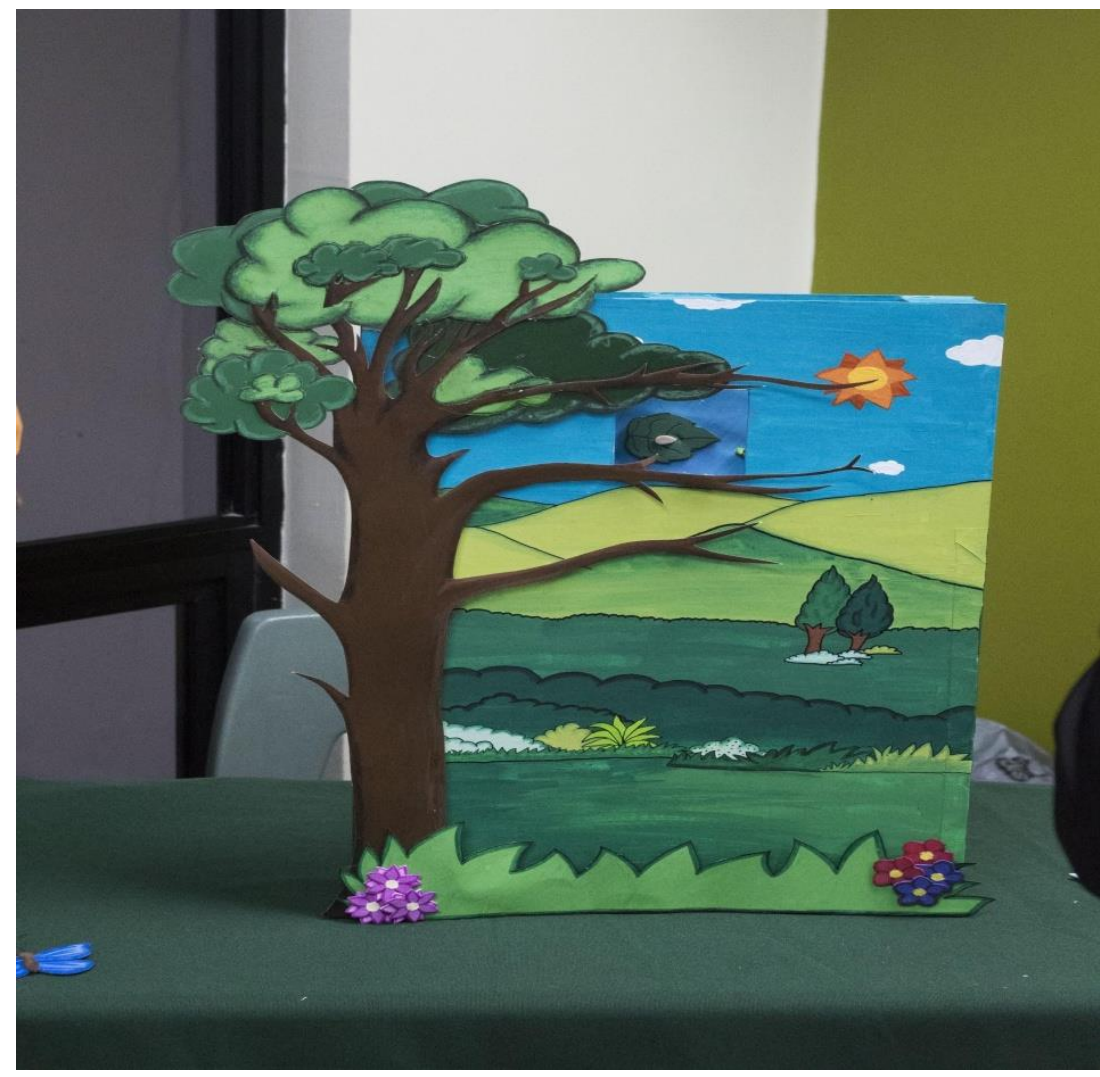

Figura 2: Prototipo de Proyecto 01

Fuente: Elaboración propia de las personas autoras, 2021.

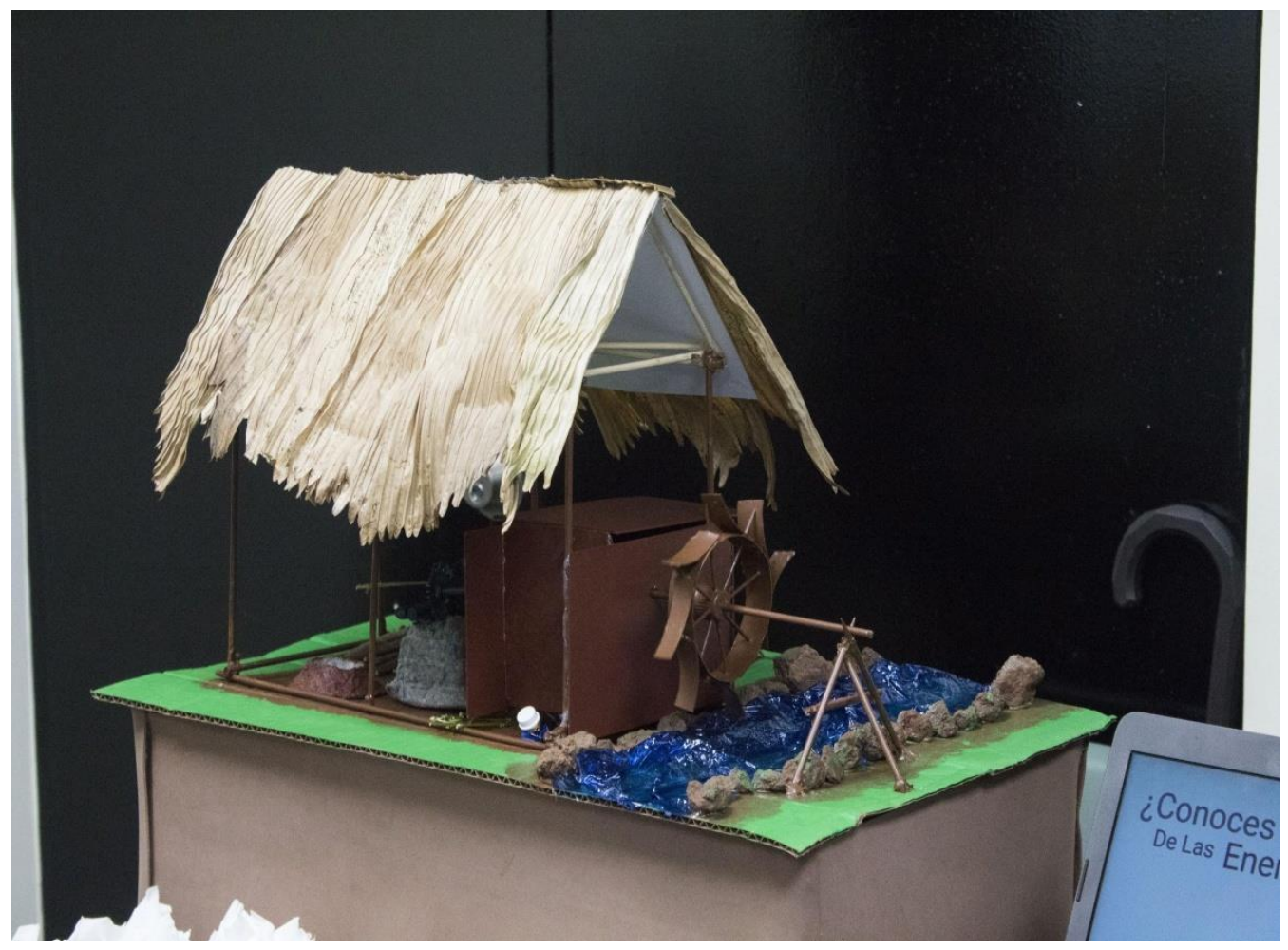

Figura 3: Prototipo de Proyecto 02 
Fuente: Elaboración propia de las personas autoras, 2021.

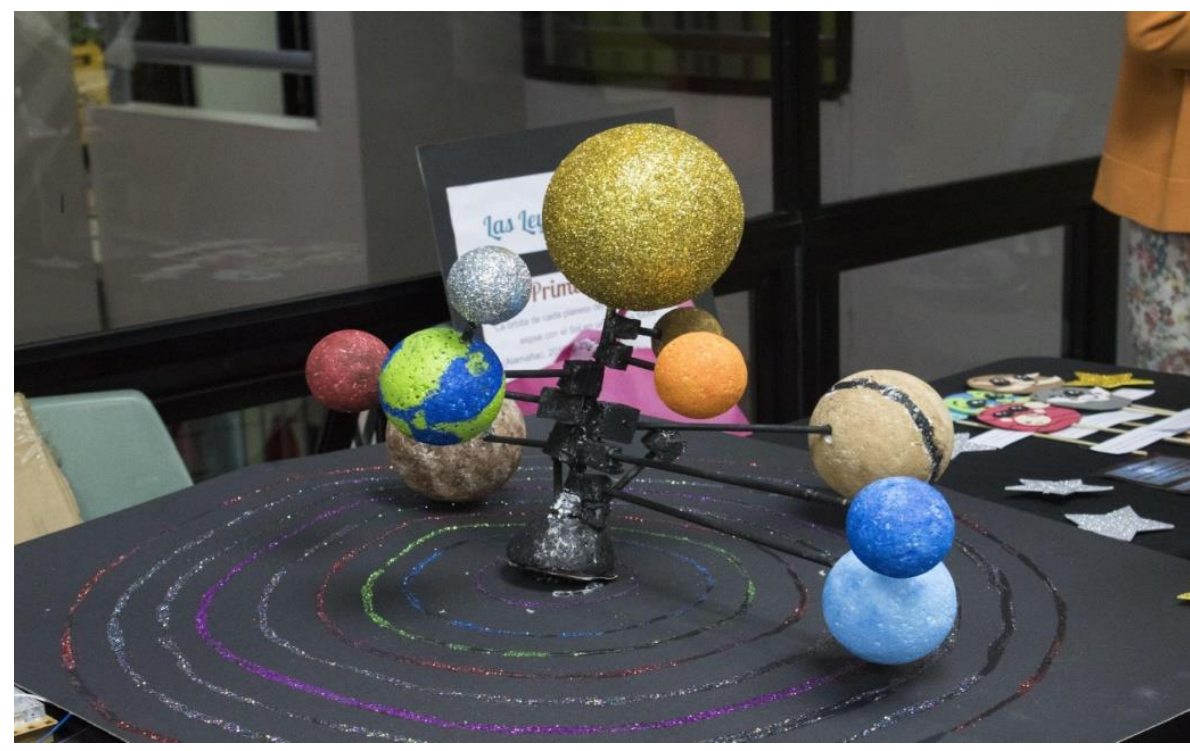

Figura 4: Prototipo de Proyecto 03

Fuente: Elaboración propia de las personas autoras, 2021.

Adicionalmente, y con el fin de abordar el tema del uso sostenible de la energía y los materiales, para la preservación y protección de los recursos del planeta, los estudiantes diseñaron una maqueta que reproduce el funcionamiento de un generador de energía eólica. Se trata de una torre con tres aspas para el viento que, al encenderse, simula el movimiento de impulso provocado por las corrientes de aire y, al mismo tiempo, enciende las luces de una casa que se encuentra alimentándose de la corriente que produce (Figura 5).

Otro de los grupos de estudiantes propuso una estrategia didáctica para enseñar los contenidos relacionados con la temática de las prácticas lectoras y la importancia de adquirir el hábito de leer. Su prototipo consiste en una plataforma que ilustra cada uno de los planetas que visita el protagonista de la obra El Principito, de Antoine de Saint-Exupéry, en el centro de dicha plataforma se encuentra una flecha en cuya punta reposa una avioneta en miniatura, cuya función es señalar cada uno de los planetas para así repasar la historia narrada en la obra (Figura 6). 


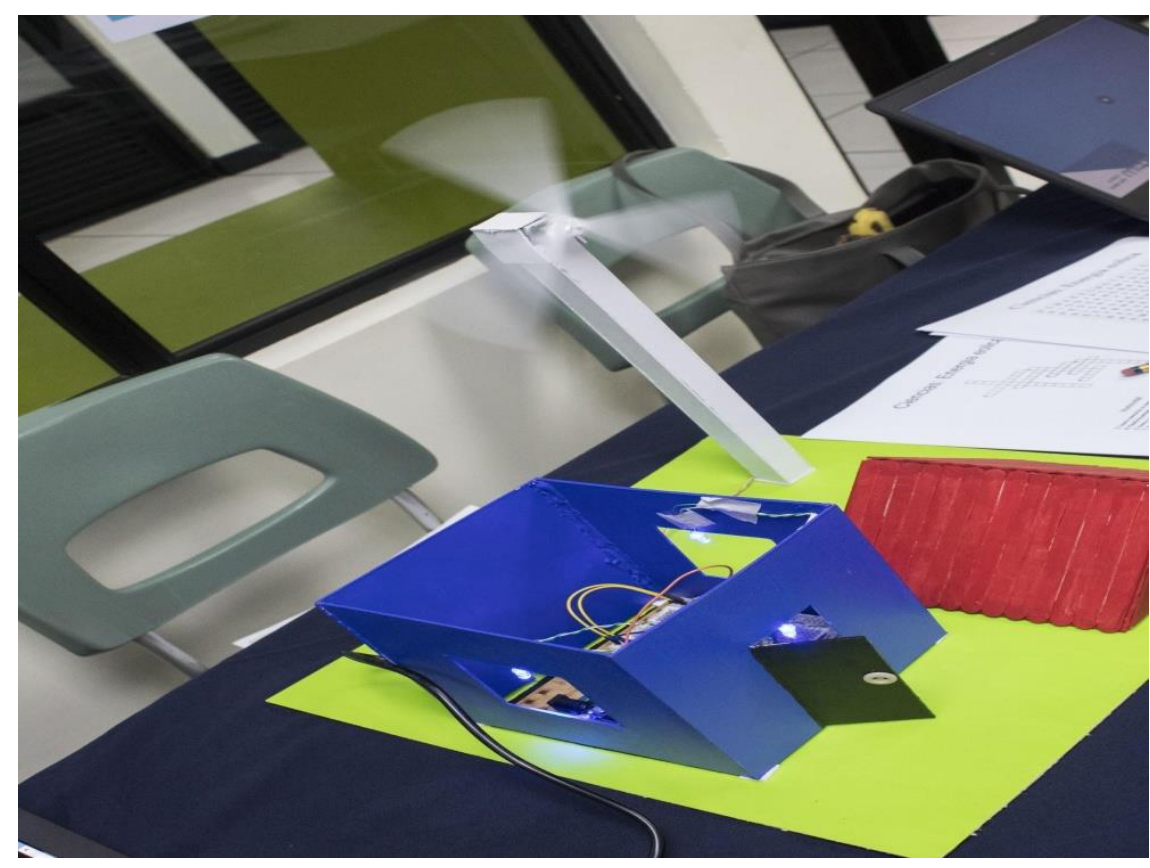

Figura 5: Prototipo de Proyecto 04

Fuente: Elaboración propia de las personas autoras, 2021.

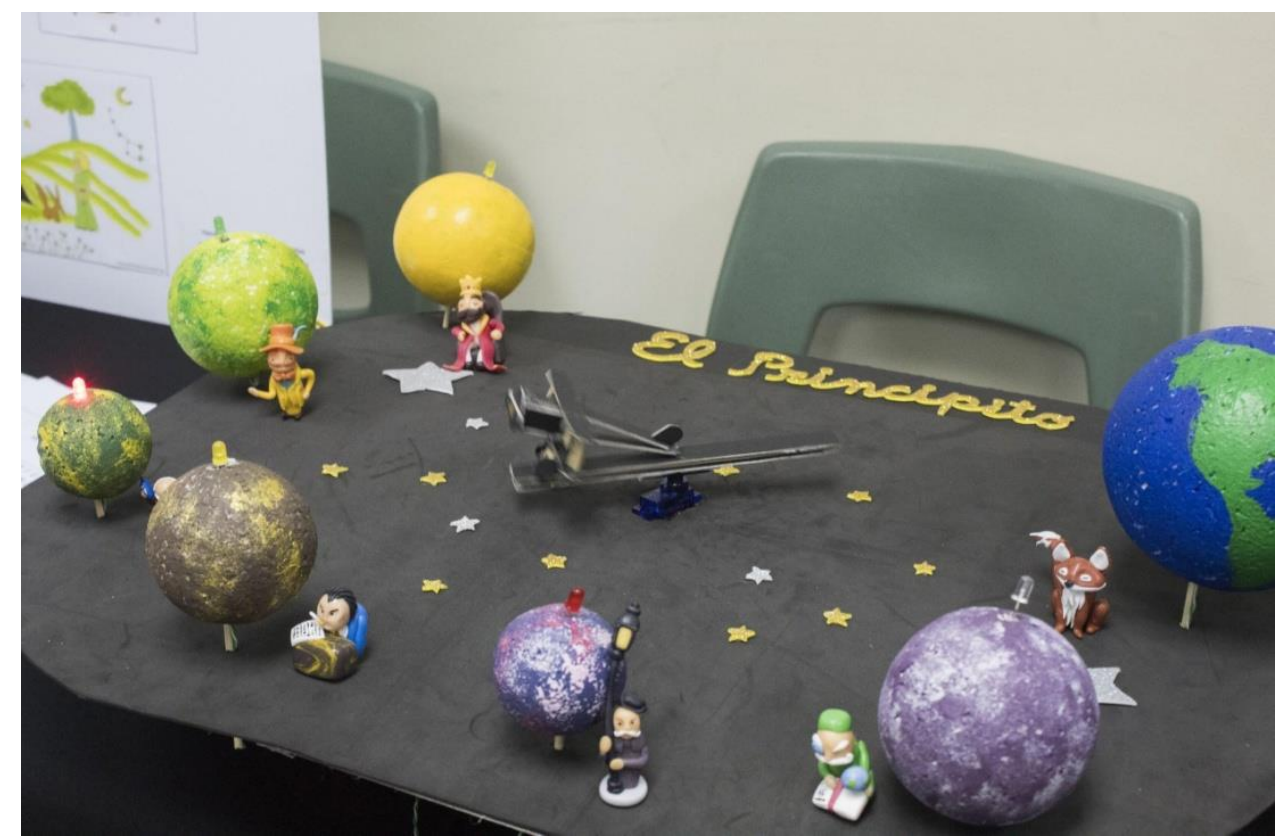

Figura 6: Prototipo de Proyecto 05

Fuente: Elaboración propia de las personas autoras, 2021.

Finalmente, para abordar la temática de Transitemos hacia una nueva cultura de Seguridad Vial, el último de los grupos de estudiantes diseñó una reproducción a escala de un cruce de dos vías para automóviles, dos cruces peatonales y sus respectivos semáforos. El funcionamiento de dicha maqueta permite enseñar el uso de los semáforos y 
la interpretación de los colores de cada luz, pues muestra de forma explícita el ciclo de luces de cada semáforo y el sonido repetitivo del semáforo peatonal (Figura 7).

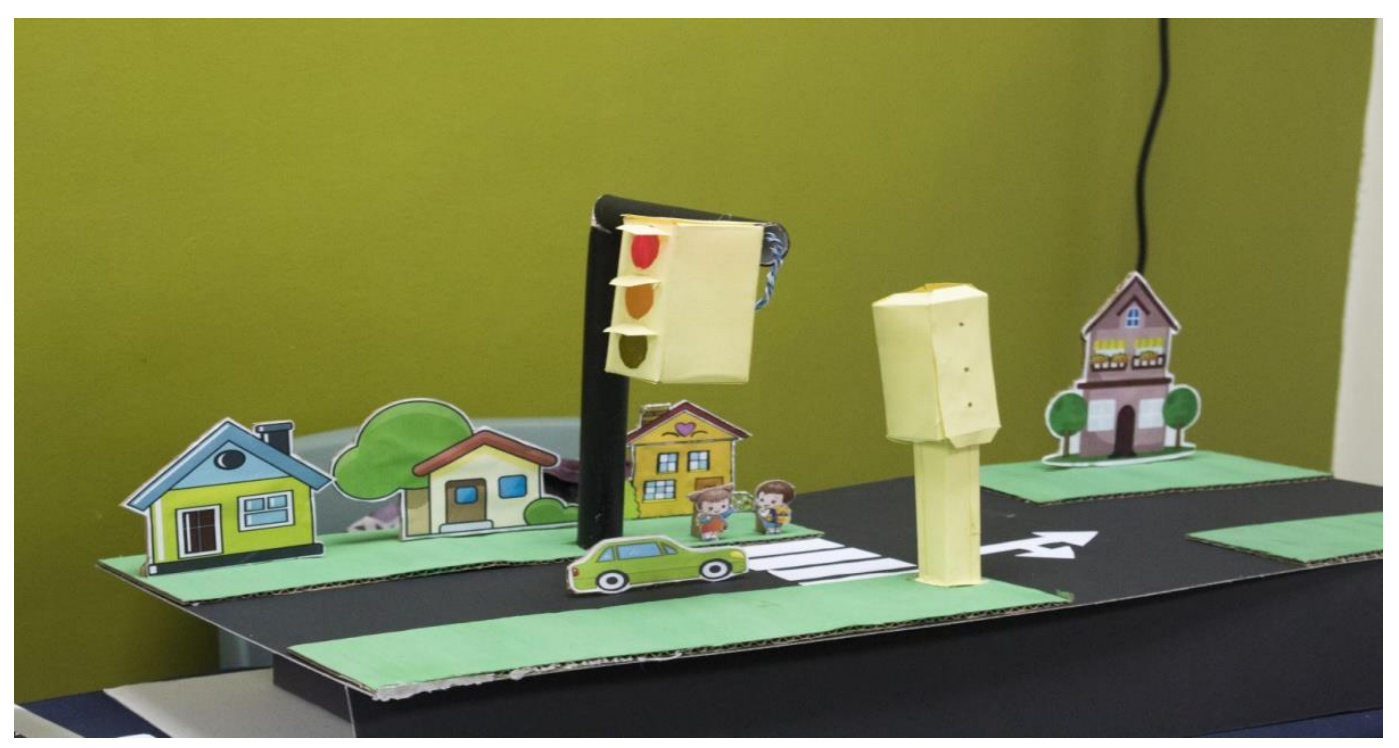

Figura 7: Prototipo de Proyecto 05

Fuente: Elaboración propia de las personas autoras, 2021.

\section{Análisis de los resultados}

Las unidades de análisis establecidas para este estudio son: la percepción de la experiencia con el diseño del material didáctico elaborado con Arduino, proceso de enseñanza - aprendizaje de la estrategia pedagógica y aportes de la experiencia en el desarrollo de habilidades en el estudiantado. En este sentido, la percepción recolectada del estudiantado participante se discute por medio del contraste con la teoría, para conocer los aspectos que conlleva el diseño de estrategias didácticas para el desarrollo de capacidades en estudiantes para el uso de hardware y software libre (Arduino y Scratch) en el diseño de proyectos educativos para la integración curricular de la biblioteca.

Para el diseño de los materiales didácticos, como se mencionó anteriormente, fue necesaria la elaboración de varios talleres, con diferentes recursos y apoyos de especialistas. Esto conllevó un proceso de planificación, coordinación y organización previa para asegurar que se contaba con las herramientas y personas calificadas. Para lograr un trabajo colaborativo e interdisciplinario para el diseño de estrategias didácticas se requiere de planificación pedagógica como "un proceso continuo y sistémico de construcción colectiva, con el propósito de organizar, diseñar, implementar, direccionar, coordinar, evaluar y sistematizar acciones y 
actividades que permitan el desarrollo del proceso de aprendizaje garantizando la transformación educativa" (MPPE, citado por Altuve, 2016, p.132).

En el caso de este estudio, la población estudiantil participante tuvo que desarrollar su propuesta en pequeños subgrupos, trabajando con el docente y el especialista en el área de tecnología, permitiendo el diseño de estrategias didácticas con Arduino, enfocadas en el cumplimiento de los objetivos planteados en el aula.

Entre los comentarios del estudiantado participante, se destacan algunos aspectos positivos como que el espacio y los recursos fueron adecuados, consideran que la estrategia les permitió crear experiencias para el fomento de la lectura de manera enriquecedora, por ejemplo: un estudiante indica que "se realizó el proyecto de energías limpias y fue una experiencia enriquecedora", otro indica que "se abordó de manera correcta". Destacan, además, la relevancia de la feria de exposición de proyectos, donde lograron exponer y observar los proyectos elaborados, lo cual consideran fue muy valioso. En este sentido, el estudiantado se manifiesta conforme con el espacio y los recursos tecnológicos, el apoyo especializado, el abordaje del facilitador y el tiempo para elaborar el material didáctico. Algunos de los comentarios de los participantes pueden apreciarse en el Cuadro 1.

\section{Cuadro 1}

Percepción de experiencia pedagógica para el diseño de material didáctico con Arduino

\begin{tabular}{|c|l|l|}
\hline \multicolumn{1}{|c|}{ Categoría } & \multicolumn{1}{|c|}{ Subcategorías } & \multicolumn{1}{|c|}{$\begin{array}{c}\text { Algunos comentarios de los } \\
\text { participantes }\end{array}$} \\
\hline $\begin{array}{l}\text { Percepción de la } \\
\text { experiencia con el } \\
\text { diseño del material } \\
\text { didáctico elaborado } \\
\text { con Arduino. }\end{array}$ & $\begin{array}{l}\text { Espacio } \\
\text { tecnológicos. Apoyo } \\
\text { especializado. técnico } \\
\text { - Abordaje del facilitador. } \\
\text { - Tiempo necesario. }\end{array}$ & $\begin{array}{l}\text { PEB1 - "Fue un espacio adecuado, } \\
\text { porque contaba con los dispositivos } \\
\text { necesarios para utilizar el Arduino } \\
\text { como estrategia didáctica". } \\
\text { PEB9 - "Se abordó de manera } \\
\text { correcta". }\end{array}$ \\
\hline
\end{tabular}

Nota: Elaboración propia de las personas autoras con base en los instrumentos aplicados a los participantes en el 2020.

Se puede inferir que el diseño de material didáctico con Arduino requiere de parte del personal docente del curso la planificación y coordinación previa para que se cuenten con 
los recursos y la capacitación necesaria para que el estudiantado pueda realizar su proyecto, además de dar seguimiento a la elaboración del material didáctico. De manera que exista un lugar, preferiblemente un espacio maker, que cuente con el equipo y el apoyo especializado para desarrollar los proyectos con Arduino.

Es relevante destacar la posición de Castrillo y Méndez (2015) en cuanto a las posibilidades de integración de la biblioteca al currículo escolar, ya que esta "se puede lograr estableciendo una relación estrecha entre la biblioteca escolar y el proyecto educativo institucional, con el fin de acompañar los proyectos de la institución y al mismo tiempo ser un espacio complementario de interacción con los diversos materiales educativos" (2015, p. 36). Asimismo, en el comentario de uno de los participantes, PEB4, "Creo que estimula el aprendizaje y además es un método didáctico espectacular que se puede usar en diferentes áreas y grados", se puede apreciar cómo existe oportunidad para que desde las bibliotecas se puedan establecer espacios más flexibles para incorporar el Arduino en la mediación pedagógica, facilitando la creación de materiales didácticos más atractivos, así como oportunidades en el enriquecimiento del proceso de aprendizaje del estudiantado.

Así mismo, en relación con el proceso de mediación pedagógica, se pueden observar algunas de las participaciones en el Cuadro 2, donde el estudiantado participante indica que estas experiencias permiten la adquisición de conocimientos de manera más atractiva, además, señalan que el Arduino permite diseñar propuestas más llamativas y atractivas. En general consideran que existe gran relevancia en aprovechar el recurso Arduino en las bibliotecas para crear proyectos educativos en su futura práctica profesional como profesionales en bibliotecología. 


\section{Cuadro 2}

Percepción del proceso de mediación de enseñanza y aprendizaje

\begin{tabular}{|c|c|c|}
\hline Categoría & Subcategorías & $\begin{array}{l}\text { Algunos comentarios de los } \\
\text { participantes }\end{array}$ \\
\hline $\begin{array}{lr}\text { Proceso de } \\
\text { enseñanza y } \\
\text { aprendizaje de } \\
\text { la estrategia } \\
\text { pedagógica. }\end{array}$ & $\begin{array}{l}\text { - Adquisición de conocimientos } \\
\text { tecnológicos de Arduino } \\
\text { (Hardware y software). } \\
\text { - Adquisición de conocimientos de } \\
\text { contenido del proyecto. } \\
\text { - Valorar las oportunidades } \\
\text { educativas del Arduino. } \\
\text { - Diseño de estrategias educativas } \\
\text { innovadoras que vinculen la } \\
\text { teórica y la práctica. }\end{array}$ & $\begin{array}{l}\text { PEB1 - "En el proceso de } \\
\text { enseñanza aprendizaje el conocer } \\
\text { el Arduino fue innovador". } \\
\text { PEB4 - "Se enseña materia teoría } \\
\text { con el quehacer práctico, es decir, } \\
\text { el aprender haciendo". } \\
\text { PEB5 -Llevar a la práctica, materia } \\
\text { teórica, estimula el desarrollo } \\
\text { cognitivo. }\end{array}$ \\
\hline
\end{tabular}

Nota: Elaboración propia de las personas autoras con base en los instrumentos aplicados a los participantes en el 2020.

Correa y Pablos (2009) establecen que son muchas las ventajas y posibilidades de las TIC, y que es necesario considerar la rentabilidad para sacar provecho a la tecnología, en este sentido contar con recursos necesarios en las bibliotecas educativas es clave para el diseño de materiales didácticos. Asimismo, los autores indican que es relevante considerar los aspectos que enmarcan su utilización en las aulas, como el papel del profesor, estudiante y manera de orientar el proceso de mediación pedagógica.

Desde esta perspectiva, el presente estudio brindó una orientación al estudiantado participante para crear su propio material didáctico acompañado de una estrategia pedagógica. Con esta experiencia se le preguntó a la población participante de estudiantes acerca de las oportunidades de incorporar el Arduino en la educación costarricense, sus respuestas se resumen en el Cuadro 3. La totalidad del estudiantado participante indicó que el Arduino tiene muchas oportunidades como parte de una estrategia didáctica en la educación. En general, de parte del estudiantado participante, se destacan muchos aportes en el desarrollo de habilidades educativas. Los aportes que señalan son los siguientes:

- El aprendizaje significativo, el desarrollo de competencias, su versatilidad para adaptarse a varias materias y grados permite la integración curricular, causa interés y permite clases más dinámicas. 


\section{Cuadro 3}

Percepción de los aportes de la experiencia en el desarrollo de habilidades

\begin{tabular}{|c|c|c|}
\hline Categoría & Subcategorías & $\begin{array}{l}\text { Algunos comentarios de los } \\
\text { participantes }\end{array}$ \\
\hline $\begin{array}{l}\text { Aportes de la } \\
\text { experiencia en } \\
\text { el desarrollo de } \\
\text { habilidades en } \\
\text { la población } \\
\text { estudiantil. }\end{array}$ & $\begin{array}{l}\text { - Clases más amenas, atractivas } \\
\text { y dinámicas para el } \\
\text { estudiantado. } \\
\text { - Posible vinculación con los } \\
\text { programas y niveles educativos } \\
\text { del MEP. } \\
\text { - Promueve el interés en el } \\
\text { aprendizaje de forma } \\
\text { significativa. } \\
\text { - Desarrollo de habilidades. } \\
\text { - Promueve la integración } \\
\text { curricular. }\end{array}$ & $\begin{array}{l}\text { PEB3 "Es enriquecedor emplear } \\
\text { Arduino en proyectos escolares, } \\
\text { debido a que el aprendizaje va a } \\
\text { ser significativo para diversos } \\
\text { estudiantes". } \\
\text { PEB4 "Ayuda a crear competencias } \\
\text { y dinamizar el proceso de } \\
\text { aprendizaje". } \\
\text { PEB5 "Creo que estimula el } \\
\text { aprendizaje y además es un } \\
\text { método didáctico espectacular que } \\
\text { se puede usar en diferentes áreas y } \\
\text { grados". }\end{array}$ \\
\hline
\end{tabular}

Nota: Elaboración propia de las personas autoras con base en los instrumentos aplicados a los participantes en el 2020.

Estos aspectos permiten valorar la incorporación del Arduino como un material que puede promover experiencias pedagógicas que fomenten el aprendizaje en los educandos. Sin embargo, queda pendiente profundizar aún más en cuáles son las habilidades o competencias que pueden mejorar o promover en la población estudiantil y en cómo este tipo de materiales didácticos con Arduino puede mejorar el aprendizaje o el desarrollo de habilidades en los educandos.

\section{Conclusiones}

La creación y aplicación de la estrategia didáctica, mediante los talleres realizados con las personas estudiantes de la Licenciatura en Bibliotecología con énfasis en Bibliotecas Educativas de la Universidad de Costa Rica, permitió, en términos generales, un acercamiento significativo al movimiento maker que está siendo muy bien recibido por las nuevas generaciones. La aplicación Scratch y la tecnología de programación en placas Arduino ofrece una alternativa innovadora y llamativa para la integración de la tecnología en el aula, permitiendo la creación de estrategias didácticas dinámicas que incentiven el 
pensamiento lógico matemático, la resolución de casos y el aprendizaje basado en problemas.

En este mismo sentido, el presente estudio permite visibilizar cómo las bibliotecas pueden convertirse en espacios de innovación que apoyen el desarrollo de proyectos didácticos, según lo cual fomenten el desarrollo de capacidades en la población estudiantil para explorar, crear y apropiarse de diferentes tecnologías disponibles en los espacios maker. Esta innovación gestada desde las bibliotecas se vincula, además, con el hecho educativo desarrollado en las aulas, esto mediante la integración curricular, por lo que la presente investigación evidencia el apoyo didáctico que puede aportar la biblioteca a las clases innovadoras en escuelas, colegios y distintos centros educativos.

Adicionalmente, se reconoce que para que la biblioteca educativa pueda convertirse en un espacio de innovación que fomente una cultura maker, debe contar con los equipos adecuados para este fin, por ejemplo: impresoras 3D, filamentos diversos, microprocesadores, computadoras, herramientas básicas de ferretería, materiales didácticos varios, entre otros. Esto es un impedimento en algunos centros educativos para realizar una réplica de dichos talleres, pero una motivación para buscarlos a través de financiamiento estatal o de otra índole.

Por otro lado, para el desarrollo de proyectos didácticos, no solo es necesario contar con equipos adecuados, sino también con personal capacitado que acompañe el proceso de diseño de los materiales didácticos con propuestas maker. Dicho proceso requiere de profesionales proactivos, con conocimientos tecnológicos y con motivación para el autoaprendizaje, la innovación, la interdisciplinariedad y el seguimiento de la actualidad, de manera que apropien a los participantes de los conocimientos básicos requeridos según el proyecto. Sin olvidar que es necesario generar espacios de colaboración en línea, en donde las diferentes participantes compartan sus productos, comenten sobre las experiencias y se genere nuevo conocimiento.

En este estudio se pudo demostrar también que es posible que el profesional de bibliotecología desarrolle proyectos didácticos con tecnologías maker vinculados con el currículo escolar, estableciendo la biblioteca en un espacio flexible e innovador que permita el 
acompañamiento y desarrollo de estos. Específicamente, la incorporación de herramientas de bajo costo como Arduino en la mediación pedagógica desde la biblioteca admite: facilitar la creación de material didáctico más creativo, desarrollar oportunidades en el enriquecimiento del proceso de aprendizaje del estudiantado y ofrecer una experiencia intuitiva a la hora de familiarizar nuevos conceptos.

Adicionalmente, la creación de un espacio para socializar los proyectos creados por las personas estudiantes no solo permite visibilizar sus trabajos, sino que además promueve una de las características más importantes del movimiento maker: el trabajo colaborativo. Mediante la Feria de Estrategias Didácticas con Microprocesadores Arduino, las personas estudiantes pudieron ver los proyectos de sus compañeros, identificar otras ideas para la creación de maquetas y circuitos y recibir retroalimentación de las personas que visitaron la Feria.

La comunidad estudiantil, por su parte, percibió la estrategia como una forma innovadora de aprender, se manifestaron satisfechos con las actividades que desarrollaron en dicha estrategia y consideraron enriquecedora la experiencia. Esto pone en evidencia que el uso de este tipo de herramientas en la creación de estrategias didácticas es bien recibido por parte de la comunidad estudiantil y les aporta nuevas competencias en las que encuentran motivación para seguir aprendiendo.

Finalmente, las estrategias didácticas en la presente investigación permiten al estudiante acercarse de una manera vivencial a los contenidos impartidos en el curso gracias a su versatilidad. El uso de herramientas como Scratch y Arduino no solo exige del estudiante una participación en la planificación de actividades dentro de la biblioteca, sino que además le permite interiorizar los contenidos mediante la resolución de problemas, incentivando su creatividad y su capacidad de análisis.

\section{Referencias}

Altuve, D. (2016). La formación docente en el proceso de la planificación pedagógica. Educ@ción en Contexto.2, 129-145. https://bit.ly/3sSGVD7

Castrillo-Sequeira, R. y Méndez-Molina, M. (2015). Integración de la Biblioteca de la Escuela La Trinidad, Moravia (San José, Costa Rica) al currículo escolar y a la 
comunidad educativa. [tesis licenciatura, Universidad Nacional de Costa Rica]. Repositorio UNA. https://bit.ly/3gEHtHE

Cobo, G y Valdivia, S. (2017). Aprendizaje Basado en Proyectos. Instituto de Docencia Universitaria (PUCP).https://bit.ly/3mG4VrL

Correa, M., y Pablos, J. (2009). Nuevas Tecnologías e innovación educativa. Revista de Psicodidáctica, 14(1), 133-145.https://bit.ly/3sULDzY

Díaz, F. (2015). Estrategias para el desarrollo de competencias en educación superior. En: Carrillo Mendoza, Gabriela (Ed), I Encuentro Internacional Universitario. El currículo por competencias en la educación superior. Ponencias y debate (pp.6386).Pontificia Universidad Católica del Perú.

Herrero-Herranz, J. y Sánchez-Allende, J. (2015). Una mirada al mundo Arduino. Revista de Ciencia, Tecnología y Medio Ambiente, (13), 3-28. https://revistas.uax.es/index.php/tec_des/article/download/617/573

Margalef, L y Arenas, A. (2006). ¿Qué entendemos por innovación educativa? A propósito del desarrollo curricular. Perspectiva Educacional, Formación de Profesores, (47), 13-31. https://bit.ly/2WvjKCL

Martí, A; Heydrich, M; Rojas, M y Hernández, A. (2010). Aprendizaje basado en proyectos: una experiencia de innovación docente. Revista Universidad EAFIT, 46(158), 1121. https://bit.ly/38i7po2

Michavila, F. (2009). La innovación educativa: Oportunidades y barreras. Revista Arbor ciencia, pensamiento y cultura. https://doi.org/10.3989/arbor.2009.extran1201

Novillo-Vicuña, J. et al. (2018). Arduino y el Internet de las Cosas. Alicante: Editorial Área de Innovación y Desarrollo. http://doi.org/10.17993/IngyTec.2018.45

Orera-Orera, L. (2008). Reflexiones sobre el concepto de biblioteca. Saberes compartidos, (2), 20-31. https://bit.ly/3gGtwJI

Paau-Cho, M.R. (2009). Viviendo el futuro en el aula: las tecnologías de la información y comunicación en los procesos de aprendizaje en la escuela primaria o básica. San José, Costa Rica: Coordinación Educativa y Cultural Centroamericana. https://bit.ly/3yw3zT2

Paredes, J; Guitert; M y Rubia, B. (2015). La innovación y la tecnología educativa como base de la formación inicial del profesorado para la renovación de la enseñanza. Revista Latinoamericana de Tecnología Educativa, 14(1), 101-114. https://bit.ly/2WwzANg 
Pritchard, S.M. (2014). Building Institutions of Knowledge: librarianship as makerspace.Libraries and the Academy, 14(4), 471-473. https://doi.org/10.1353/pla.2014.0030

Randi, J. y Corno, L. (2000). Los profesores corno innovadores. En Goodson, I.F., Good, T. y Biddle, B.J. (Coord.), La enseñanza y los profesores (pp. 169-236). Paidós.

Rodríguez, E., Vargas, É. y Luna, J. (2010). Evaluación de la estrategia "aprendizaje basado en proyectos". Revista Educación y Educadores, 13(1), 13-25. https://bit.ly/3zliZKV

Sancho, J. M. y Alonso-Cano, C. (Coord.) (2012). La fugacidad de las políticas, la inercia de las prácticas: la educación y las tecnologías de la información y la comunicación. Octaedro.

Seas-Tencio, J. (2016). Didáctica General I. EUNED.

Sheehy, K., Ferguson, R., y Clough, G. (2014). Augmented Education: Bringing Real and Virtual Learning Together (Digital Education and Learning). Palgrave Macmillan.

Stallman, R. (2004). Software libre para una sociedad libre. Editorial Traficantes de Sueños.

Tellez-Ramírez, M. (2016). Incursión de la robótica en la educación. Investigación y tecnología, 4(2), 41-52. https://bit.ly/3DqFOPW

Valverde-Berrocoso, J., Fernández-Sánchez, M.R. y Garrido-Arroyo, M.C. (2015). El pensamiento computacional y las nuevas ecologías del aprendizaje. RED - Revista de Educación a Distancia, 46(3), 1-18. https://bit.ly/38ilZuw

Valverde-Chavarría, J. (2005). Software libre, alternativa tecnológica para la educación. Revista Electrónica "Actualidades Investigativas en Educación”, 5(2), 1-9. https://bit.ly/3BkWzdn

Willging, P., Astudillo, G. y Bast, P. (2010). Aprender a programar (¿y a pensar?) jugando. https://bit.ly/2Y7mVRN

Yockey, M. y Donovan, M.A. (2015). Maker Spaces. Illinois Reading Council Journal, 43(3), 56-60.

Zaltman, G; Duncan, R. y llolbek, J. (1973). Innovations and Organizations. J. Wiley \& Sons. 


\section{Notas de las personas autoras}

Hazel Castro-Araya. Profesora en la Carrera de Educación Primaria. de la Universidad de Costa Rica y Coordinadora del Programa de Tecnologías Educativas para el Aprendizaje (PROTEA). Correo electrónico: hazel.castroaraya@ucr.ac.cr, ORCID: https://orcid.org/0000-0003-1875-5770

Daniela Esquivel-Calderón. Bibliotecóloga en la Unidad de Automatización del Sistema Nacional de Bibliotecas, Ministerio de Cultura y Juventud, Costa Rica. Correo electrónico: desquivelc@sinabi.go.cr, ORCID:https://orcid.org/0000-0002-6536-1814

Jairo Guadamuz-Villalobos. Profesor en la Escuela de Bibliotecología y Ciecias de la Información de la Universidad de Costa Rica. Correo electrónico: jairo.guadamuz@ucr.ac.cr, ORCID:https://orcid.org/0000-0002-0284-3656 